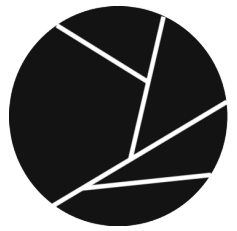

VISUAL SPACES OF CHANGE: DESIGNING INTERIORITY

SHELTER, SHAPE, PLACE, ATMOSPHERE

ISSN: 2183-8976 [PRINT] 2183-9468 [ONLINE]

Volume 5, Issue 1| Publication year: 2020

DOI 10.24840/2183-8976_2020-0005_0001_9

(c) SCOPIO EDITIONS

HOMEPAGE:

\title{
Light Catcher
}

\section{by Mark Durden}

Among his remarkable performance-based short films made in the garden of his family home, two films show the artist holding a mirror to both catch and reflect sunlight back to the camera and viewer. Such performances provide a fitting allegory for his relationship to the medium of photography. As a photographer Peter Finnemore is someone who catches and plays with light.

Light is key to the pictures made in his home place in rural mid Wales, Gwendraeth House. The photographs relay the intimacy he has with his childhood home, which has been in his family for generations. Finnemore has been photographing his home for thirty years and his pictures are full of hints and suggestions, traces of those who live and lived there. With people's passing, he is now its sole occupant and the house has become more and more a portrait of his own imagining, his dream space.

Finnemore photographs feelingly and describes his home as "a dreaming centre to divine and survey the spaces between darkness and stars". Working with black and white film and the chemical-based printing process his richly toned prints explore the opposition and gradations between non-light and light, negative and positive, with all their symbolic implications. Like film, the house and its rooms are seen as receptive and responsive spaces. In Dream Traces a partly decorated wall above a bed is animated both by the gestural traces of darker paint upon it and lighter rectangular areas where posters and pictures were once attached. The wall is not 
blank but a field of different energy forces, the slow photographic effect of the darkening of the wall around the absent pictures against the more immediate brushmarks of house paint at its edges. The wall is also suggestive of an awakening state, the sense of something not fully coming into consciousness. This is in contrast to the relative order and geometry introduced by the wooden bars of the bedstead and the clarity of the singing and piercing detail of the white dot at the centre of an eye, painted on glass. This Greek mati, used to ward off evil, becomes the focal point of this picture and cue to many objects and elements in his pictures that are felt to be imbued with energies and powers beyond their material form. 
The house, its furnishing and objects are humble but they become special and magical in these pictures. In Wallpaper Fragrance, the shadow of a face falls upon flowers printed on wallpaper. Above this fugitive portrait is a framed aerial photograph of the home and its plot of land. The interior opens to the exterior, a sense of the world beyond the home and a sense of the home's place within the world. The house, set within its quite extensive grounds, is close to and part of the natural world. Concentrating on a layering and interplay of surface patterns and imprints in his pictures, there is an abundance of floral imagery in these interiors. Such décor is not seen or judged in terms of class or assumptions of what is in good or bad taste. The floral prints and patterns on wallpaper, carpets, curtains, cushions, as well as the ornamental animal figures in Finnemore's pictures, all take on a symbolic dimension. In conveying the presence and continuity of the surrounding natural world into the home they suggest a natural life force, energy and process. In a remarkable picture of a dying black cat that appears to be turning to shadow, the fecundity of the floral imagery the cat is set amidst counters the darkness sucking out the light and life.

While there can be moments of darkness there is much light in these pictures: light flooding and illuminating the upstairs rooms or dappling darkened walls and also present symbolically in the recurrence of such radiant forms as shawls and sunflowers and the pervasive floral patterning that runs throughout these pictures. Finnemore as light catcher has gifted us some of the most distinctive, beautiful and lyrical responses to interior spaces in the medium of photography. 


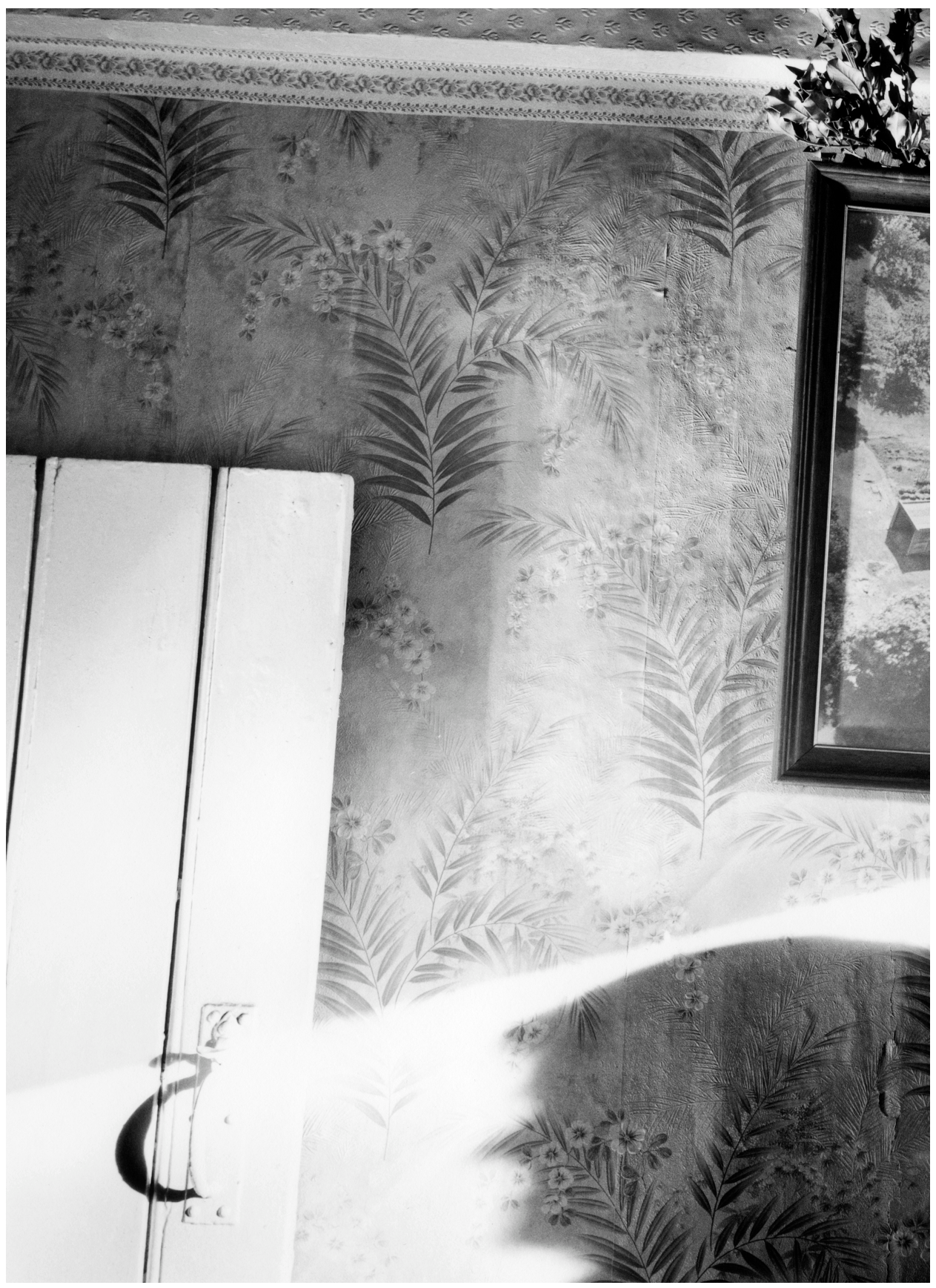



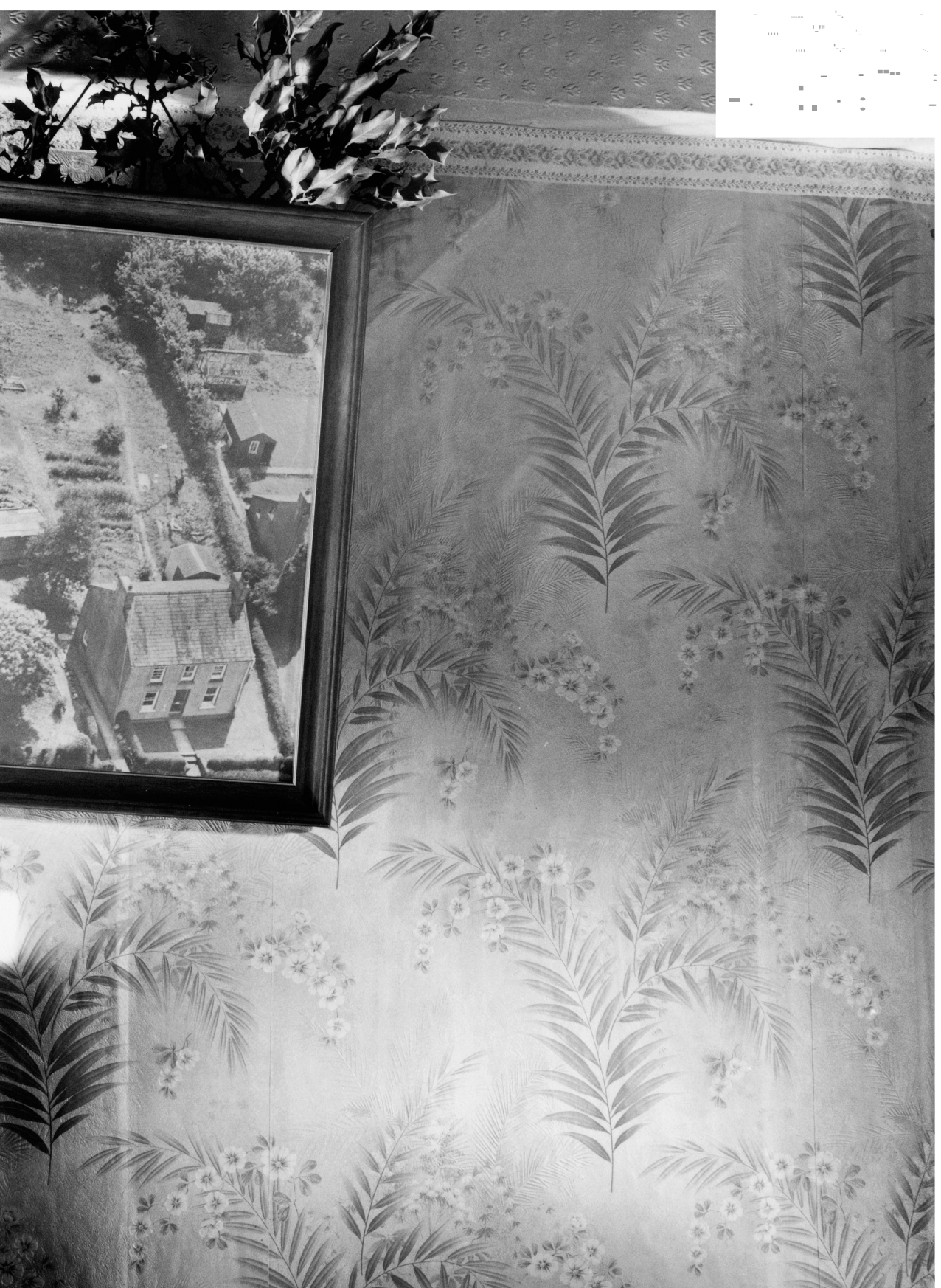

$\sqrt{2}$

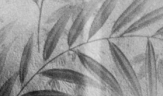

$=$

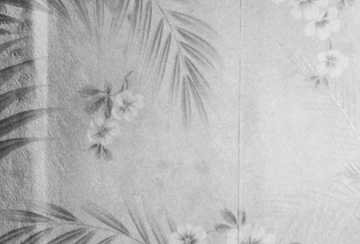

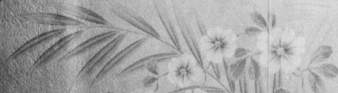
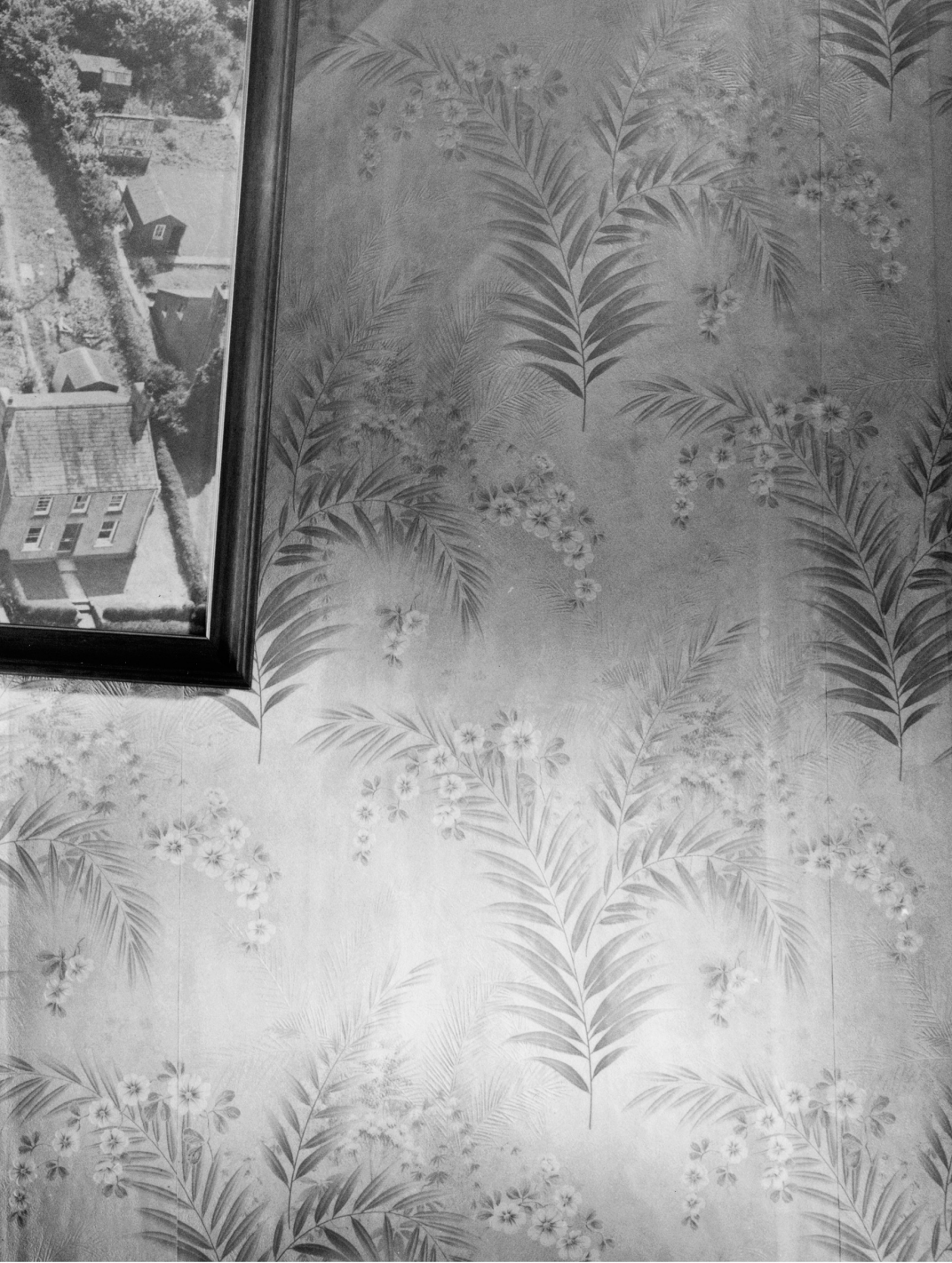

Tr

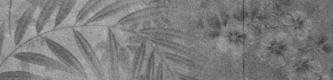

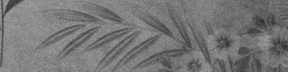

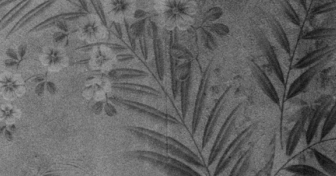




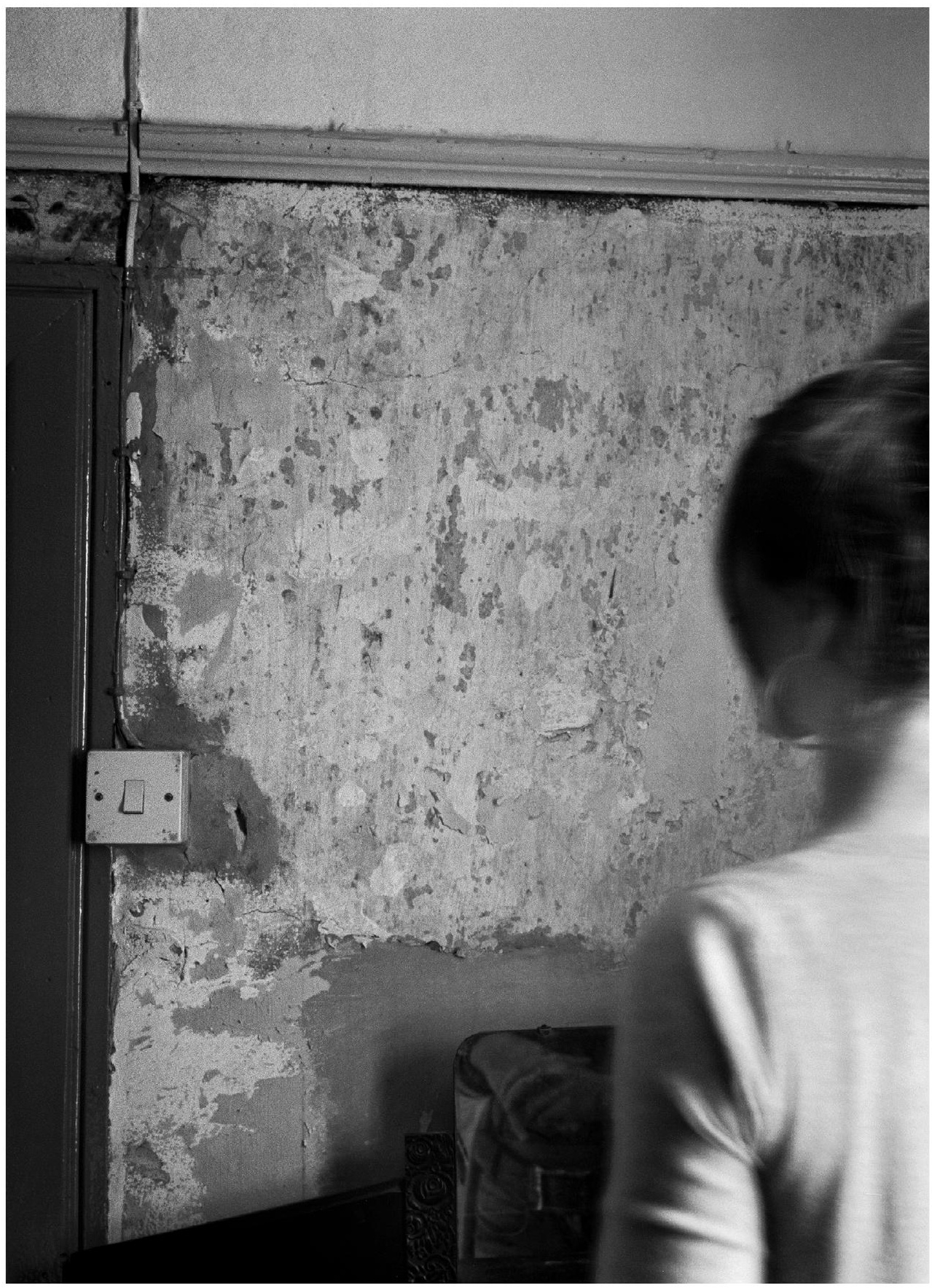




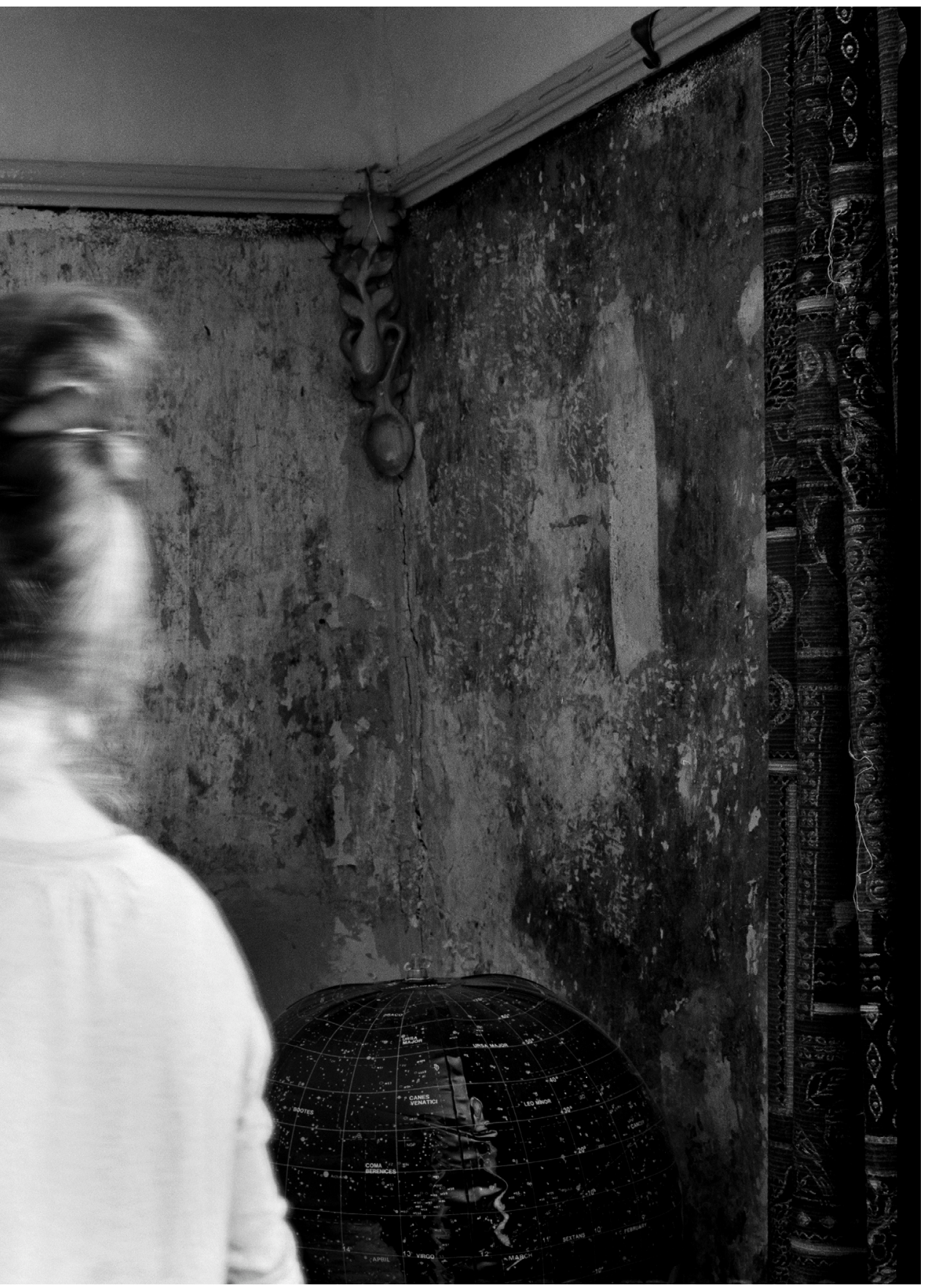

